\title{
Automatic Strengthening of Graph-Structured Knowledge Bases
}

\author{
Vinay Chaudhri, Nikhil Dinesh, Stijn Heymans, Michael Wessel ${ }^{\dagger}$ \\ \{ vinay.chaudhri | nikhil.dinesh | stijn.heymans | michael.wessel\}@sri.com \\ SRI International \\ Artificial Intelligence Center \\ 333 Ravenswood Avenue \\ Menlo Park, California 94025-3493, USA \\ $\left({ }^{\dagger}=\right.$ corresponding author $)$
}

\begin{abstract}
We address two problems in underspecified graphstructured knowledge bases (GSKBs): the coreference and the provenance problem. Both problems are important for a variety of reasons. The former asks "Which existentially quantified variables in different but related axioms of a GSKB possibly denote identical domain individuals?", and the latter "From which axioms in a GSKB is a piece of knowledge getting derived?" To decide the former, we need to be able to prove equality between different variables - a GSKB in which this is possible is called a strenghtened GSKB, and an underspecified GSKB otherwise. The latter occur naturally in many knowledge acquisition contexts, and are also easier to author. We hence present an algorithm which rewrites an underspecified GSKB into a strengthened GSKB, by virtue of Skolemization and addition of equality atoms such that the coreference information can be drawn from it. This enlarges the logical theory (the deductive closure) of the GSKB and strengthens its inferential power, hence affecting the provenance information. Our algorithm is model-theoretic in nature and exploits a novel class of desirable, preferred models, which capture the desired co-references. The algorithm is a logical reconstruction of an implemented algorithm that we successfully applied to a large-scale biological knowledge base, in which it identified more that 22,000 equality atoms.
\end{abstract}

\section{Introduction}

Graph-structured underspecified knowledge bases occur naturally, for example, if biological knowledge is modelled graphically by virtue of concept graphs as in the AURA project [Gunning, D. and Chaudhri, V. K. et al., 2010], see Fig. 1, or if knowledge is formulated in natural language:

S1 Every Cell has part a Ribosome and a Chromosome.

S2 Every EukaryoticCell is a Cell.

S3 Every EukaryoticCell has part a EukaryoticRibosome, a EukaryoticChromosome, a Nucleus, such that the Eukaryotic Chromosome is inside the Nucleus.
S4 Every EukaryoticRibosome is a Ribosome.

S5 Every EukaryoticChromosome is a Chromosome.

Here the question arises - is the Eukayotic Chromosome that $S 3$ is talking about actually the Chromosome from $S 1$ ? Such assumptions are often reasonable. We are calling a knowledge base which does not answer this question definitely underspecified. These kinds of question are studied to some extent in the field of computational linguistics under the term anaphora resolution [Carpenter, 1994], [Cohen, 2007]. We will use co-reference resolution in the following.

From a logical point of view, these sentences correspond to FOPL formulas (the comma in consequents denotes conjunctions, and we combine $S 2, S 3$ into $S 23$ ):

$$
\begin{aligned}
& \text { S1 } \forall x: \operatorname{Cell}(x) \Rightarrow \exists x_{1}, x_{2} \text { : } \\
& \text { hasPart }\left(x, x_{1}\right) \text {, Ribosome }\left(x_{1}\right) \text {, } \\
& \text { hasPart }\left(x, x_{2}\right) \text {, Chromosome }\left(x_{2}\right) \\
& \text { S23 } \forall x: \text { EukaryoticCell }(x) \Rightarrow \exists x_{3}, x_{4}, x_{5}: \operatorname{Cell}(x) \text {, } \\
& \text { hasPart }\left(x, x_{3}\right) \text {, Euk.Ribosome }\left(x_{3}\right) \text {, } \\
& \text { hasPart }\left(x, x_{4}\right) \text {, Euk.Chromosome }\left(x_{4}\right) \text {, } \\
& \text { hasPart }\left(x, x_{5}\right), \operatorname{Nucleus}\left(x_{5}\right), \text { inside }\left(x_{4}, x_{5}\right) \\
& \text { S4 } \forall x: \text { Euk.Ribosome }(x) \Rightarrow \text { Ribosome }(x) \\
& \text { S5 } \forall x: \text { Euk.Chromosome }(x) \Rightarrow \text { Chromosome }(x)
\end{aligned}
$$

In the following, unary predicates are called concepts, and binary predicates relations. The concept $D$ is called a superconcept of $C$ if $\forall x: C(x) \Rightarrow D(x), \ldots$ holds, and consequently, $C$ is called a subconcept of $D$.

Co-references are, in general, tedious or even impossible to specify at knowledge authoring time (e.g., if the input is underspecified in the first place) and hence, an automatic coreference resolution algorithm is often desirable. It will necessarily have to rely on some sort of guessing or hypothetical (logically unsound) reasoning. Related motivation and mechanisms can be found in the literature, e.g., in the reasoning system "Knowledge Machine" (KM) [Clark and Porter, 1997] a so-called unification operator is employed for this purpose. A KM-like unification operator has also been studied in the context of underspecified object-oriented knowledge bases formalized in Answer Set Programming (ASP) [Chaudhri and Tran, 2012]. These and related approaches (e.g., based on Description Logics) are discussed in more detail in Section 5.

We consider co-reference resolution in GSKBs an important problem to solve because of its potential to reduce mod- 


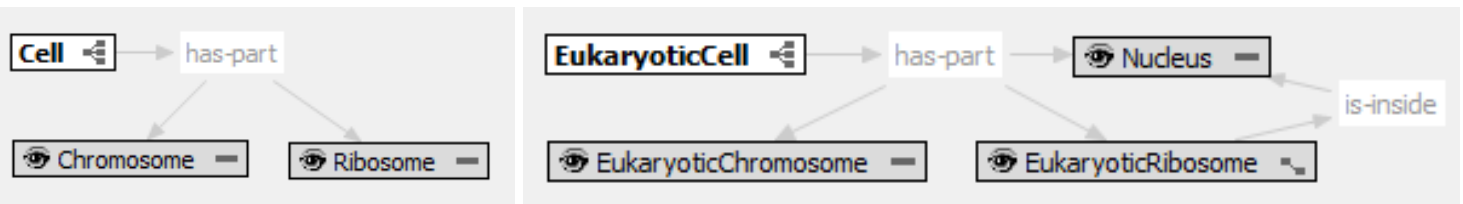

Figure 1: (Simplified) Concept Graphs for Cell and EukaryoticCell in AURA.

eling effort. For example, if we extend Ribosome $\left(x_{1}\right)$ in $S 1$ by saying that it is inside $\operatorname{Cytosol}\left(x_{6}\right)$, resulting in

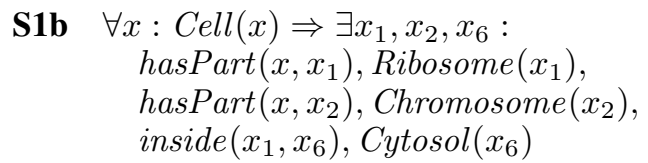

then we would like to derive that this also holds for the Euk.Ribosome $\left(x_{3}\right)$ in EukaryoticCell - it is reasonable to assume that the Euk.Ribosome got inherited from Cell as a Ribosome which then got specialized. However, this requires that $x_{3}$ in $S 23$ and $x_{1}$ in $S 1 b$ are co-referential. We can enforce this as follows, using Skolem functions and equalities:

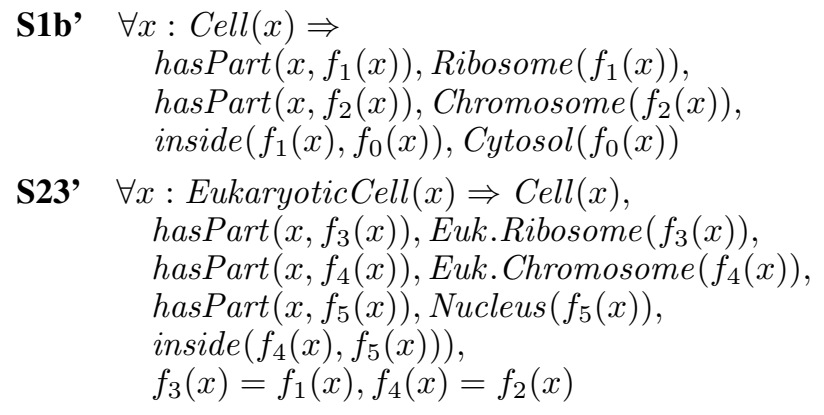

In this strengthened $G S K B$, we have $\left\{S 1 b^{\prime}, S 23^{\prime}, S 4, S 5\right\} \models$ $\forall x: \operatorname{EukaryoticCell}(x) \Rightarrow \exists x_{3}, x_{6}: \operatorname{hasPart}\left(x, x_{3}\right)$, Euk.Ribosome $\left(x_{3}\right)$, inside $\left(x_{3}, x_{6}\right)$, Cytosol $\left(x_{6}\right)$,

as desired, due to $f_{3}(x)=f_{1}(x)$ in $S 23^{\prime}$. Note that this does not hold in the underspecified original GSKB: $\{S 1 b, S 23, S 4, S 5\} \quad \forall \forall x \quad \forall$ : EukaryoticCell $(x) \quad \Rightarrow \quad \exists x_{3}, x_{6} \quad: \quad \operatorname{hasPart}\left(x, x_{3}\right)$, Euk.Ribosome $\left(x_{3}\right)$, inside $\left(x_{3}, x_{6}\right)$, Cytosol $\left(x_{6}\right)$. Hence, inside $\left(x_{3}, x_{6}\right)$ would be redundant (implied) if added to $S 23^{\prime}$ in the strengthened GSKB, but would not be redundant if added to the underspecified original GSKB. This shows that the provenance of an atom is affected by the co-references. Redundant / implied atoms can be removed from the GSKB, keeping its size smaller and more managable, simplifying also retraction (if each atom is local / asserted).

The contribution of this paper is the presentation of a novel GSKB rewriting algorithm. It rewrites a GSKB such as $\{S 1 b, S 23, S 4, S 5\}$ into a strengthened GSKB, similar to $\left\{S 1 b^{\prime}, S 23^{\prime}, S 4, S 5\right\}$. From the strengthened GSKB we compute the provenance of atoms and co-references. We will use a model theoretic notion of preferred models to characterize the additional desirable inferences that we wish to get from the underspecified GSKB. The information in the preferred model is used to rewrite a Skolemized version of the GSKB into a strengthened version of the GSKB, and its models are preferred - they include at least the preferred model. Obviously, deciding entailment of atoms, and hence the prove- nance problem, are in general undecidable in $\operatorname{FOPL}(=)$, but decidable in the considered fragment.

The paper is structured as follows: We first formally define the GSKB framework and required notions of GSKB and strengthened GSKB, and the semantic notion of preferred models. We then present the algorithm and show that the strengthened GSKB (produced by the algorithm) has models which are preferred which hence gain the required additional conclusions in order to decide the provenance and co-reference problems. Next we evaluate the algorithm on a large-scale biological graph-structured GSKB from the AURA project [Gunning, D. and Chaudhri, V. K. et al., 2010]. Finally we conclude and discuss related and future work.

\section{Graph Structured Knowledge Bases}

In the following, we denote an atom or a conjunction of atoms with free variables $\left\{x, x_{1}, \ldots, x_{n}\right\}$ as $\varphi(x, \vec{x})$, with $\vec{x}=$ $\left(x_{1}, \ldots, x_{n}\right)$. Graph-structured knowledge bases (GSKBs) are formulated in first order-logic with equality, $\operatorname{FOPL}(=)$. We assume that there is a function terms which returns the terms in a formula, e.g. $\operatorname{terms}\left(R\left(t_{1}, t_{2}\right)\right) \triangleq\left\{t_{1}, t_{2}\right\}$ :

Definition 1. Basic Definitions. Let $\mathcal{C}(\mathcal{R})$ be a countably infinite set of unary (binary) predicate names, and $\mathcal{F}=$ $\left\{f_{1}, f_{2}, \ldots\right\}$ be a countably infinite set of unary function names - hence, $(\mathcal{C} \cup \mathcal{R}, \mathcal{F})$ is the signature. Elements in $\mathcal{C}(\mathcal{R})$ are called concepts (relations). Moreover, let $\mathcal{X}=$ $\left\{x, x_{1}, x_{2}, \ldots\right\}$ be a countably infinite set of variables. A GSKB term is a term $t$ such that $t \in \mathcal{X}$, or $t=f_{i}(x)$, or $t=f_{i}\left(f_{j}(x)\right)$, with $\left\{f_{i}, f_{j}\right\} \subseteq \mathcal{F}$. Let $t, t_{1}, t_{2}$ be GSKB terms:

GSKB atoms: Let $\{C, D\} \subseteq \mathcal{C}, R \in \mathcal{R},\{v, w\} \subseteq \mathcal{X}$. Then, $C(v)$ and $C\left(f_{i}(x)\right)$ are concept atoms, and $R(v, w)$, $R\left(x, f_{i}(x)\right)$ are relation atoms. Moreover, there are equality and inequality atoms of the following form: $f_{i}(x)=f_{j}(x), f_{i}(x)=f_{j}\left(f_{k}(x)\right), f_{j}\left(f_{k}(x)\right)=f_{i}(x)$, and $f_{i}(x) \neq f_{j}(x)$, with $i, j, k$ pairwise unequal.

GSKB rule: For a concept $C$, a formula $\rho_{C} \triangleq \forall x$ : $C(x) \Rightarrow \exists ! \vec{x}: \varphi(x, \vec{x})$ is called a GSKB rule, where $\varphi(x, \vec{x})=\bigwedge_{i \in 1 \ldots m} \alpha_{i}$ is finite conjunction of GSKB atoms. This is shorthand for $\forall x$ : $C(x) \Rightarrow \exists \vec{x}$ : pairwise_unequal $(x, \vec{x}) \wedge \varphi(x, \vec{x}), \vec{x}=$ $\left(x_{1}, \ldots, x_{n}\right)$, with the macro pairwise_unequal $(x, \vec{x}) \triangleq$ $\bigwedge_{1 \leq i<j \leq n} x_{i} \neq x_{j} \wedge \bigwedge_{1 \leq i \leq n} x_{i} \neq x$.

For a concept $C$ with $\rho_{C}=\forall x: C(x) \Rightarrow \exists ! \vec{x}$ : $\varphi(x, \vec{x})$, denote $\varphi(x, \vec{x})=\bigwedge_{i \in 1 \ldots m} \alpha_{i}$ as a set by $\tau_{C} \triangleq$ $\left\{\alpha_{1}, \ldots, \alpha_{m}\right\}$, and terms $(C) \triangleq \bigcup_{\alpha \in \tau_{C}} \operatorname{terms}(\alpha)$.

We require that the terms in terms $\left(\rho_{C}\right)$ are connected to $x:$ for all $t \in \operatorname{terms}(C)$, connected $(x, t)$ holds, where 
connected is defined as follows: connected $\left(t_{1}, t_{2}\right)$ holds if $\left\{R\left(t_{1}, t_{2}\right), R\left(t_{2}, t_{1}\right)\right\} \cap \tau_{C} \neq \emptyset$, or there is some $t$ s.t. connected $\left(t_{1}, t\right)$ and connected $\left(t, t_{2}\right)$ holds.

GSKB: A finite set of GSKB rules $\Sigma$ in which there is at most one rule per concept.

Input GSKB: $A$ GSKB which is function-free and without equality atoms.

Auxiliary notions: Given a GSKB $\Sigma$, we refer to the set of concepts used in $\Sigma$ as concepts $(\Sigma)$, and $\tau_{C, \Sigma}$ to refer to the consequent of $\rho_{C} \in \Sigma$. We extend the other definitions to accept a $\Sigma$ argument as well, e.g., terms $(C, \Sigma)$, etc.

For example, $\{S 1 b, S 23, S 4, S 5\}$ is an (underspecified) input GSKB, and $\left\{S 1 b^{\prime}, S 23^{\prime}, S 4, S 5\right\}$ is a strengthened (output) GSKB; however, we need to replace the $\exists$ quantifier with $\exists$ !. The formal definition of strengthened GSKB is given below. Note that sometimes the strengthening algorithm will not add anything, and hence output will equal input, e.g. for $\{S 4, S 5\}$.

We require that an input GSKB must be coherent:

Definition 2. Coherent GSKB and coherent model. A GSKB $\Sigma$ is coherent if there is standard first-order model $\mathcal{I}=$ $\left(\Delta_{\mathcal{I}},{ }^{\mathcal{I}}\right), \mathcal{I}=\Sigma$, in which every concept $C$ mentioned in $\Sigma$ is interpreted in a non-empty way: $C^{\mathcal{I}} \neq \emptyset$. Such a model is called a coherent model.

Moreover, we define standard notions such as superconcepts as follows:

Definition 3. Auxiliary Definitions. Let $C$ be a concept, $\Sigma$ be a GSKB. We then define the following functions and predicates w.r.t. $\Sigma$ :

- asserted_types $(C, \Sigma) \triangleq\left\{D \mid D(t) \in \tau_{C, \Sigma}\right\}$

- has_asserted_type $(C, D)$ iff $D \in$ asserted_types $(C, \Sigma)$

- asserted_superconcepts $(C, \Sigma) \triangleq\left\{D \mid D(x) \in \tau_{C, \Sigma}\right\}$

- has_asserted_superconcept ${ }_{\Sigma}(C, D)$ iff $D \in$ asserted_superconcepts $(C, \Sigma)$

- superconcepts $(D, \Sigma)$

$$
\triangleq\left\{E \mid \text { has_asserted_superconcept } t_{\Sigma}^{+}(D, E)\right\}
$$

- has_superconcept $t_{\Sigma}(C, D)$ iff $D \in$ superconcepts $(C, \Sigma)$

- all $_{-} \operatorname{types}_{\Sigma}(C)$

$$
\begin{aligned}
& \triangleq\{E \mid D \in \text { asserted_types }(C, \Sigma) \text {, } \\
& E \in \text { superconcepts }(D, \Sigma)\} \\
& \cup \text { superconcepts }(C, \Sigma)
\end{aligned}
$$

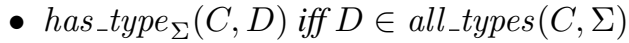
where $R^{+}$denotes the transitive closure of relation $R$.

We require that the relations has_superconcept $t_{\Sigma}$ and has_type $_{\Sigma}$ are irreflexive and define:

Definition 4. Admissible GSKB. An input GSKB $\Sigma$ is called admissible if $\Sigma$ is coherent, has_superconcept $t_{\Sigma}$ and has_type ${ }_{\Sigma}$ are irreflexive, and if there are no implied concept atoms in the rules: for all $C \in$ concepts $(\Sigma)$, if $D(t) \in \tau_{C, \Sigma}$, then for all $E \in$ superconcepts $(D, \Sigma): E(t) \notin \tau_{C, \Sigma}$.

The following is straightforward:
Proposition 1. Every admissible GSKB $\Sigma$ has a coherent, finite model.

Proof. Given that we do not support negation of concepts or relations, and given that inequality atoms are only introduced by the $\exists$ ! quantor, inconsistencies such as $x \neq x$ cannot occur. Moreover, since GSKB has_superconcept $t_{\Sigma}$ and has_type $e_{\Sigma}$ are irreflexive, the GSKB is acyclic, and the consequent of every rule can be "unfolded", analog to the unfolding of an acyclic TBox in description logics [Baader et al., 2003]. This produces a finite consequent for every rule. Next, for every $\rho_{C} \in \Sigma, C$ can be instantiated s.t. $i_{C} \in C^{\mathcal{I}}$ holds, and we can easily satisfy the existentials in the consequent by creating one instance per variable. The process terminates and produces a model of $\Sigma$ which is coherent and finite.

We need a notion of connectedness on models:

Definition 5. Predicate connected on models. Let $\mathcal{I}=$ $\left(\Delta_{\mathcal{I}},{ }^{\mathcal{I}}\right)$ be a model of $\Sigma$. For $i, j \in \Delta_{\mathcal{I}}$, we define connected $_{\mathcal{I}}(i, j)$ if, for some $R \in \mathcal{R},\{(i, j),(j, i)\} \cap R^{\mathcal{I}} \neq$ $\emptyset$, or there is some $k \in \Delta_{\mathcal{I}}$ s.t. connected con $_{\mathcal{I}}(i, k)$ and connected $_{\mathcal{I}}(k, j)$.

In the following we are considering admissible GSKBs only, and we are interested in their preferred models. The intuition behind the notion of a preferred model is the following: for every concept $C$, there should be a prototypical model of $C$ which is not also a model of some non-superconcept of $C$, in the form of a connected graph that "mirrors" the atoms in $\tau_{C, \Sigma}-$ due to the pairwise_unequal macro there will be at least one individual per variable in $\rho_{C}$ in this model. Moreover, the prototypical model for $C$ also contains inherited "graphs" from concepts in all_types ${ }_{\Sigma}(C)$. Hence, the graph satisfying the atoms $\tau_{C, \Sigma}$ is only a subgraph of the full model for $C$. Most importantly, the notion of a preferred model captures the intuition that inherited content can be specialized, and hence should give rise to co-references: in the prototypical model for EukaryoticCell, the Chromosome inherited from its superclass Cell will be represented by the same individual as its own local Euk.Chromosome. Note that this minimizes the extension of Chromosome. The same argument applies to arbitrary conjunctions: we will not identify the inherited Chromosome with the local Euk.Ribosome, as this would result in a model in which Chromosome $\wedge$ Euk.Ribosome is interpreted non-empty, and there are models in which this conjunction is interpretated by the empty set. These intuitions are formalized as follows:

Definition 6. Preferred model of admissible GSKB $\Sigma$. Let $\Sigma$ be an admissible $G S K B$, and $\mathcal{I}=\Sigma$ be a coherent finite model. Then, $\mathcal{I}$ is called preferred if the following holds:

1. for every concept $C \in$ concepts $(\Sigma)$, there is (at least) one $i \in C^{\mathcal{I}}$ s.t. for all $D$, if has_superconcept $(D, C)$, then $i \notin D^{\mathcal{I}}$ - hence, there is at least one element which is "unique" to $C$, and denoted by $i_{C}$.

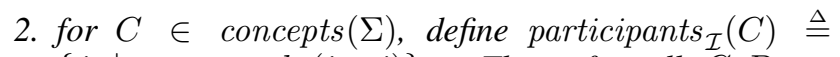
$\left\{j \mid\right.$ connected $\left._{\mathcal{I}}\left(i_{C}, j\right)\right\}$. Then, for all $C, D \in$ concepts $(\Sigma)$, with $C \neq D$, the following holds: participants $_{\mathcal{I}}(C) \cap$ participants $_{\mathcal{I}}(D)=\emptyset$. 
3. for every non-empty subset $\mathcal{C S} \subseteq$ concepts $(\Sigma)$, there is no preferred model $\mathcal{I} \neq \mathcal{I}^{\prime}$, with $\Delta_{\mathcal{I}^{\prime}} \subseteq \Delta_{\mathcal{I}}$ s.t. $\bigcap_{C \in \mathcal{C S}} C^{\mathcal{I}^{\prime}} \subset \bigcap_{C \in \mathcal{C S}} C^{\mathcal{I}}$.

Consider the preferred models of $\{S 1, S 23, S 4, S 5\}$. We are forced to have at least one "unique" Cell which is not a EukaryoticCell, due to 1 . Otherwise, every Cell would acquire the properties of EukaryoticCells, which is not desirable. Moreover, none of the individuals connected to that unique $C e l l$ are shared by another concept, due to 2 . Hence, the concept models have the forms of "non-overlapping graphs", and inherited content is "mapped in". We are forced to minimize the extension of every concept, as well as of every conjunction of concepts. This prevents models in which, for example, Ribosome $\mathcal{I}^{\mathcal{I}} \cap$ Euk. Chromosome $e^{\mathcal{I}} \neq \emptyset$ holds, as there are smaller models in which they are interpreted disjointly: Ribosome $e^{\mathcal{I}} \cap$ Euk. Chromosome ${ }^{\mathcal{I}}=\emptyset$. Note that the inequality atoms in $\Sigma$ only prevent "merging" of variables within the same formula, but the individual for Chromosome $\left(x_{2}\right)$ inherited from Cell could in principle be made co-referential with the local Euk.Ribosome $\left(x_{3}\right)$ in EukaryoticCell. This is prevented in a preferred model. Also, looking at the model of EukaryoticCell, the coreference between the from Cell inherited Chromosome $\left(x_{2}\right)$ and its own local Euk.Chromosome $\left(x_{4}\right)$ is made explicit, since this will result in the smallest (extension of) Chromosome $e^{\mathcal{I}}$. A model in which a EukaryoticCell would have two different Chromosomes would be larger and in violation to 3 . So, we only make those conjunction true in a preferred model that we have to make true - for example, Cell $^{\mathcal{I}} \cap$ EukaryoticCell ${ }^{\mathcal{I}} \neq \emptyset$, due to $S 23$, and there is no model in which this conjunction is interpreted by a smaller set.

Note that a preferred model is not a "minimal" model in the classical sense. Consider $\forall x: C(x) \Rightarrow$ $\exists ! x 1: R(x, x 1), D(x 1), \forall x \quad: \quad S u b C(x) \Rightarrow \exists ! x 2 \quad$ : $C(x), R(x, x 2), E(x 2)$. In the classical minimal model $\mathcal{I}$, we would have $\# \Delta_{\mathcal{I}}=2$, and it would satisfy $D \wedge E$. Also, $C^{\mathcal{I}}=S u b C^{\mathcal{I}}$. But this is not what we want. It violates 1,2 , as well as 3 . The preferred model will need at least 5 nodes.

In principle, there can be more than one preferred model and hence, more than one strengthened version of the GSKB. For example, consider the GSKB

$$
\begin{aligned}
C(x) \Rightarrow \exists ! x_{1}: R\left(x, x_{1}\right), & E\left(x_{1}\right) \\
\operatorname{SubC}(x) \Rightarrow \exists ! x_{2}, x_{3}: & C(x), \\
& R\left(x, x_{2}\right), E\left(x_{2}\right), F\left(x_{2}\right), \\
& R\left(x, x_{3}\right), E\left(x_{3}\right), G\left(x_{3}\right) .
\end{aligned}
$$

Here, $x_{1}$ in $C$ can be co-referential with either $x_{2}$ in $S u b C$, or with $x_{3}$.

In the next section, we will show the following constructively, by specifying an algorithm which constructs a preferred model for a given admissible GSKB $\Sigma$ :

Proposition 2. Every admissible GSKB has a preferred model.

We can now state the purpose of the GSKB strengthening algorithm more clearly. Given an admissible GSKB $\Sigma$ (note that this is an input GSKB), we are interested in finding a strengthened version of $\Sigma$ :
Definition 7. Strengthened version of $\Sigma$. Given an admissible (input) GSKB $\Sigma$, we are calling $\Sigma^{\prime}$ a strengthened version of $\Sigma$ if the following holds:

1. for every rule $\rho_{C} \in \Sigma$, there is a rule $\rho_{C}^{\prime} \in \Sigma^{\prime}$ that uses only the variable $x$ : terms $\left(\rho_{C}^{\prime}\right) \cap \mathcal{X}=\{x\}$.

2. if $\mathcal{I}^{\prime} \models \Sigma^{\prime}$ is a standard first-order model of $\Sigma^{\prime}$ which is coherent, then $\mathcal{I}^{\prime} \models \Sigma$, and $\mathcal{I}^{\prime}$ is a preferred model for $\Sigma$. Hence, $\Sigma^{\prime} \models \Sigma$.

From a strengthened GSKB, we can decide provenance and co-reference as follows:

Definition 8. Provenance and co-reference determination. Let $C$ be a concept, $\Sigma^{\prime}$ be a strengthened GSKB, and $\mathcal{P} \subseteq$ $\tau_{C, \Sigma^{\prime}}$. With $\beta=\bigwedge_{\alpha \in \mathcal{P}} \alpha$, we then say that $\beta$ (and hence all the atoms in $\mathcal{P})$ are

- local (or asserted) in $C$ if

$$
\begin{gathered}
\Sigma^{\prime} \backslash\left\{\rho_{C}\right\} \cup\left\{\forall x: C(x) \Rightarrow \bigwedge_{\alpha \in \tau_{C, \Sigma^{\prime}} \backslash \mathcal{P}} \alpha\right\} \\
\forall \forall \forall x: C(x) \Rightarrow \beta,
\end{gathered}
$$

- and inherited otherwise. More specifically, $\beta$ (and $\mathcal{P}$ ) is inherited from $D$, iff $D(t) \in \tau_{C, \Sigma^{\prime}}$, and $\beta^{\prime}=$ $\bigwedge_{\alpha \in \mathcal{P}^{\prime}} \alpha$ with $\mathcal{P}^{\prime}=\left\{\alpha_{\left[f_{i}(t) \Rightarrow f_{i}(x)\right]} \mid \alpha \in \mathcal{P}\right\}$ is local in $D$, and there is no more general SupD with has_superconcept $t_{\Sigma^{\prime}}(D, S u p D)$ such that $\beta($ and $\mathcal{P})$ is inherited from SupD.

Moreover, given concepts $C, D$, two GSKB terms $t_{1} \in$ terms $(C), t_{2} \in \operatorname{terms}(D)$ are said to be co-referential in $\Sigma^{\prime}$ iff either $t_{1}=t_{2}=x, t_{1}=f_{i}(x), t_{2}=f_{j}\left(f_{k}(x)\right)$, or $t_{2}=f_{i}(x), t_{1}=f_{j}\left(f_{k}(x)\right)$, and $\Sigma^{\prime}=(\forall x: C(x) \Rightarrow$ $f_{i}(x)=f_{j}(x) \vee\left(\forall x: D(x) \Rightarrow f_{i}(x)=f_{j}(x)\right)$.

Note that a conjunction $\beta$ is local as soon as some atom is already local. Hence, if a complex conjunction $\beta$ (resp. $\mathcal{P}$ ) is local, this does not mean that all its atoms have to be local some atoms may be inherited.

Proposition 3. Provenance and co-reference are decidable in a strengthened GSKB $\Sigma^{\prime}$.

The proof is given in the next Section.

\section{Constructing a Strengthened GSKB}

The algorithm works by performing the following steps:

1. Produce the skolemized version of $\Sigma, \Sigma_{S}$, by bringing every rule in $\Sigma$ into Skolem normal form. The Skolemized axioms contain no nested function terms, only terms of the form $f_{i}(x)$ and $x$. Let $\mathcal{O} \triangleq$ $\left\{o_{C} \mid C \in \operatorname{concepts}(\Sigma)\right\}$ be a set of constants, and also add $\left\{C\left(o_{C}\right) \mid C \in\right.$ concepts $\left.(\Sigma)\right\}$ to $\Sigma_{S}$.

2. Construct the minimal Herbrand model $\mathcal{I}_{\mathcal{H}}=$ $\left(\Delta_{\mathcal{H}},{ }^{I_{\mathcal{H}}}\right)$ of $\Sigma_{S}$. The minimal Herbrand model is unique and finite, given that $\Sigma$ is admissible (and does not contain disjunctions in the consequents). Note that the minimal Herbrand model will automatically satisfy the inequality atoms, and it will also satisfy points 1 and 2 from Definition 6, due to the set of constants $\mathcal{O}$ which are instantiated as $\left\{C\left(o_{C}\right) \mid C \in\right.$ concepts $\left.(\Sigma)\right\} \subseteq \Sigma_{S}$, and with the exception of $x$, there are no shared terms in the rules of $\Sigma_{S}$, as Skolemization creates fresh function symbols for every variable. Thus, $o_{C}$ represents the 
root individual of the unique model for concept $C$, with $o_{C}^{\mathcal{I}_{\mathcal{H}}}=i_{C}, i_{C} \in C^{\mathcal{I}_{\mathcal{H}}}$.

3. Transform $\mathcal{I}_{\mathcal{H}}$ into a preferred model of $\Sigma, \mathcal{I}_{\mathcal{A}}=$ $\left(\Delta_{\mathcal{A}},{ }^{\mathcal{I}_{\mathcal{A}}}\right) . \quad \Delta_{\mathcal{A}}$ is the quotient set of $\Delta_{\mathcal{H}}$ under the $=$ equivalence relation, $\Delta_{\mathcal{A}}=\Delta_{\mathcal{H}} \backslash=$. Hence, the elements of $\Delta_{\mathcal{A}}$ represent the equivalence classes of equated Skolem ground terms from the Herbrand universe $\Delta_{\mathcal{H}}$. This step is non-deterministic, as there may be more than one preferred model for $\Sigma$.

4. Use $\mathcal{I}_{\mathcal{A}}$ to construct a strengthened GSKB $\Sigma^{\prime}$ from $\Sigma_{S}$ which is satisfied by that model. Use the equivalent clusters in $\Delta_{\mathcal{A}}$ to generate equality atoms.

5. From $\Sigma^{\prime}$ it is possible to decide the provenance and the co-reference problem, on a syntactic basis.

Since steps 1 and 2 are standard and well-know [Hedman, 2004], let us define the algorithm for step 3. We need two more utility notions before we can proceed:

Definition 9. Relations $\mathcal{E}$ and $\mathcal{U}$, and equivalence classes. Let $\mathcal{I}_{\mathcal{H}}=\left(\Delta_{\mathcal{H}},{ }^{I_{\mathcal{H}}}\right)$ be the minimal unique Herbrand model after step 2 of $\Sigma_{S}$ above. Let $\mathcal{E}$ be a binary relation over terms from the Herbrand universe $\Delta_{\mathcal{H}}$, and define $\operatorname{closure}(\mathcal{E}) \triangleq \bigcup_{C \in \operatorname{concepts}}(\Sigma), k \in \Delta_{\mathcal{H}}$ $\left\{\left(f_{1}(k), f_{2}(k)\right) \mid\left(f_{1}\left(o_{C}\right), f_{2}\left(o_{C}\right)\right) \in \mathcal{E}^{\circledast}\right\} \cup$

$\left\{\left(f_{1}\left(f_{2}(k)\right), f_{3}(k)\right) \mid\left(f_{1}\left(f_{2}\left(o_{C}\right)\right), f_{3}\left(o_{C}\right)\right) \in \mathcal{E}^{\circledast}\right\} \cup$

$\left\{\left(f_{1}\left(f_{2}(k)\right), f_{3}\left(f_{4}(k)\right)\right) \mid\left(f_{1}\left(f_{2}\left(o_{C}\right)\right), f_{3}\left(f_{4}\left(o_{C}\right)\right)\right) \in \mathcal{E}^{\circledast}\right\}$ and.$\circledast$ denotes the reflexive, symmetric, and transitive closure of a relation. Let $[i]^{\mathcal{E} \triangleq} \triangleq\{j \mid(i, j) \in \operatorname{closure}(\mathcal{E})\}$. Moreover, let $\mathcal{U} \triangleq\left\{[i]^{\mathcal{E}} \neq[j]^{\mathcal{E}} \mid i_{1} \in[i]^{\mathcal{E}}, j_{1} \in[j]^{\mathcal{E}}, C \in\right.$ concepts $\left.(\Sigma),\left(i_{1} \neq j_{1}\right) \in \tau_{C, \Sigma_{S}}\right\}$ be the set of inequality atoms.

Intuitively, $(i, j) \in \mathcal{E}$ represents $i=j$, and $[i]^{\mathcal{E}}$ represents the equivalence class of $i$. The relation $\mathcal{E}$ (and hence the equivalence classes) will grow as pairs of equated individuals / terms are added by the algorithm given below. Intuitively, the closure operator makes sure that whenever two terms starting from the same root node $o_{C}$ are equated in the unique model of $C$, that then this equality will also hold for all its $C$ instantiations in other parts of the model. Note that also $\mathcal{U}$ will grow, representing inferences such as $i \neq j, k \neq l, j=k \Rightarrow i \neq l$.

The algorithm can now be stated as follows:

Algorithm 1. Construction of a preferred model for $\Sigma$. Let $\mathcal{I}_{\mathcal{H}}=\left(\Delta_{\mathcal{H}},{ }^{I_{\mathcal{H}}}\right)$ be the minimal unique Herbrand model of $\Sigma_{S}$ after step 2 above.

1. define hasRoot $(i) \triangleq o_{C}$ iff connected ${ }_{\mathcal{I}_{\mathcal{H}}}\left(o_{C}, i\right)$ holds, for every $C \in$ concepts $(\Sigma)$.

2. then, non-deterministically apply the following merging rule on the model as long as it is applicable:

if there are individuals $i, j \in \Delta_{\mathcal{H}}, i \neq j$,
with hasRoot $(i)=$ hasRoot $(j)=o_{C}$ and
ind_types $(i) \subseteq$ ind_types $(j), i \notin[j]^{\mathcal{E}}$,
$[i]^{\mathcal{E}} \neq[j]^{\mathcal{E}} \notin \mathcal{U}$, then $\mathcal{E} \triangleq \mathcal{E} \cup\{(i, j)\}$.

Assume the rule application stops with a global maxi-

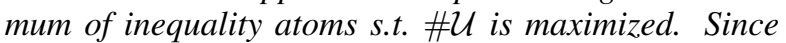
this is a non-deterministic algorithm, such a run exists, and we can assume that the non-deterministic algorithm will produce it.

3. define $\mathcal{I}_{\mathcal{A}}=\left(\Delta_{\mathcal{A}},{ }^{\mathcal{I}_{\mathcal{A}}}\right)$ as follows:

$\Delta_{\mathcal{A}} \triangleq\left\{[i]^{\mathcal{E}} \mid i \in \Delta_{\mathcal{H}}\right\}$, and for all $C \in$ concepts $(\Sigma)$ : $C^{\mathcal{I}_{\mathcal{A}}} \triangleq\left\{[i]^{\mathcal{E}} \mid i \in C^{\mathcal{I}_{\mathcal{H}}}\right\}$, for all $R \in \mathcal{R}: R^{\mathcal{I}_{\mathcal{A}}} \triangleq$ $\left\{\left([i]^{\mathcal{E}},[j]^{\mathcal{E}}\right) \mid(i, j) \in R^{\mathcal{I}_{\mathcal{H}}}\right\}$.

The algorithm terminates, since $\mathcal{I}_{\mathcal{H}}$ is finite, so there is a finite number of merging possibilities in the rule. The solution which maximizes $\# \mathcal{U}$ can obviously be found by search in a deterministic version.

Lemma 1. $\mathcal{I}_{\mathcal{A}}=\left(\Delta_{\mathcal{A}},{ }^{\mathcal{I}_{\mathcal{A}}}\right)$ is a preferred model for $\Sigma$.

Proof. Obviously, $\mathcal{I}_{\mathcal{A}}$ is finite and coherent, as it was constructed by the algorithm based on the unique finite Herbrand model. Assume that $\mathcal{I}_{\mathcal{A}}$ is not a preferred model for $\Sigma$. By construction, $\mathcal{I}_{\mathcal{A}}$ is a model of $\Sigma_{S}$, as the merging rule preserves the model character of $\mathcal{I}_{\mathcal{H}}$. Since $\mathcal{I}_{\mathcal{H}}$ is a model of the Skolemized version, it is also a model of $\Sigma$, since $\Sigma_{S} \models \Sigma$ for the Skolemized GSKB [Hedman, 2004]. Hence, $\mathcal{I}_{\mathcal{A}}$ is a model of $\Sigma$, also.

It remains to show that it is preferred. Assume that it is not. Since points 1 and 2 from Definition 6 are already satisfied by construction, only 3 can be violated. Then, there must be some other model $\mathcal{I}^{\prime}$ and some $\mathcal{C S} \subseteq$ concepts $(\Sigma)$ such that $\bigcap_{C \in \mathcal{C S}} C^{\mathcal{I}^{\prime}} \subset \bigcap_{C \in \mathcal{C S}} C^{\mathcal{I}}$, witnessed by $[i]^{\mathcal{E}} \in \bigcap_{C \in \mathcal{C S}} C^{\mathcal{I}}$ with $[i]^{\mathcal{E}} \notin \bigcap_{C \in \mathcal{C S}} C^{\mathcal{I}^{\prime}}$.

1. If $\bigcap_{C \in \mathcal{C S}} C^{\mathcal{I}^{\prime}}=\emptyset$, then this violates the assumption that $\Sigma_{\mathcal{H}}$ was a minimal Herbrand model (which does not make things true without need). Hence, $\bigcap_{C \in \mathcal{C} S} C^{\mathcal{I}}=\emptyset$ as well, which contradicts $[i]^{\mathcal{E}} \in \bigcap_{C \in \mathcal{C S}} C^{\mathcal{I}}$.

2. Assume $\mathcal{C S}=\{D\}$ is a single concept name. As $\mathcal{I}_{\mathcal{H}}$ was a minimal model, the existence of $i$, with $i \in[i]^{\mathcal{E}}$, is somehow enforced by $\Sigma_{S}$, hence there is some term $t_{i} \in \operatorname{terms}\left(C, \Sigma_{S}\right)$ with $D \in i_{\text {ind_types }}\left(t_{i}\right)$. Moreover, for the same reason, $D^{\mathcal{I}^{\prime}} \neq \emptyset$, as otherwise it wouldn't be a model, but $i \notin D^{\mathcal{I}^{\prime}}$. Consequently, there is some $j \in D^{\mathcal{I}^{\prime}}$ with $i \neq j$. Then, there must also be some $t_{j} \in$ terms $\left(C, \Sigma_{S}\right)$ with $D \in$ ind_types $\left(t_{j}\right)$, with $t_{i} \neq t_{j}$.

There are a couple of cases:

(a) Assume ind_type $\left(t_{i}\right) \subseteq$ ind_types $\left(t_{j}\right)$

i. if $C^{\prime}=C$ and hence $\operatorname{hasRoot}(i)=$ $\operatorname{hasRoot}(j)=C$, then $\left(t_{i} \neq t_{j}\right) \notin \tau_{C, \Sigma_{S}}$ and $[i]^{\mathcal{E}} \neq[j]^{\mathcal{E}} \notin \mathcal{U}$, as otherwise $\mathcal{I}$ would not be a model. But then, the merging rule would have been applied and merged $i$ and $j$, such that $[i]^{\mathcal{E}}=[j]^{\mathcal{E}}=\{i, j\}$. Rule application could not have been blocked by the precondition $[i]^{\mathcal{E}} \neq[j]^{\mathcal{E}} \notin \mathcal{U}$, because $\mathcal{I}_{\mathcal{A}}$ was produced by a run in which $\# \mathcal{U}$ was maximized. This means that the rule will be applicable and equate $i$ and $j$, contradicting the assumption that the algorithm has terminated.

ii. otherwise, $C \neq C^{\prime}$, then we don't have to worry: as stated in Definition 6, participants ${ }_{\mathcal{I}_{\mathcal{A}}}(C) \cap$ participants $_{\mathcal{I}_{\mathcal{A}}}\left(C^{\prime}\right)=\emptyset$. 
(b) Assume ind_type $\left(t_{j}\right) \subseteq$ ind_types $\left(t_{i}\right)$ : analog to the previous case.

(c) Assume ind_type $\left(t_{i}\right) \nsubseteq$ ind_types $\left(t_{j}\right)$. Then there is some $E \in$ ind_type $\left(t_{i}\right), E \notin$ ind_types $\left(t_{j}\right)$. As $\mathcal{I}_{\mathcal{A}}$ was a minimal Herbrand model, and there is no way for $[i]^{\mathcal{E}}$ to "vanish" from $E^{\mathcal{I}_{\mathcal{A}}}$, there must be $[i]^{\mathcal{E}} \in E^{\mathcal{I}_{\mathcal{A}}}$ and hence $[i]^{\mathcal{E}} \in \bigcap_{C \in \mathcal{C S}} C^{\mathcal{I}^{\prime}}$. Contradiction.

3. If $\mathcal{C S}=\left\{D_{1}, \ldots, D_{n}\right\}$, then there must already be some $\mathcal{C S}^{\prime}=\left\{D_{m}, D_{n}\right\}, \mathcal{C S}^{\prime} \subseteq \mathcal{C S}$ for which we have such an $i$. If has_superconcept $\left(D_{m}, D_{n}\right)$ or vice versa, then there is already some $\mathcal{C} \mathcal{S}^{\prime}=\left\{D_{m}\right\}$, and this is handled by 2. Otherwise, $D_{m}, D_{n}$ are not in a sub/superconcept relationship, and corresponding instances are not getting merged by the merging rule. But similar to 2c), this will lead us to conclude that $[i]^{\mathcal{E}} \in \bigcap_{C \in \mathcal{C S}} C^{\mathcal{I}^{\prime}}$, contradicting the assumption.

Hence, $\mathcal{I}_{\mathcal{A}}$ is a preferred model. Note that this proves Proposition 2.

For what remains to be shown is how we can compute a strengthened GSKB from $\Sigma_{S}$ and $\mathcal{I}_{\mathcal{A}}$.

Definition 10. Construction of strengthened GSKB $\Sigma^{\prime}$. Let $\Sigma_{S}$ be the skolemized version of the admissible GSKB, and $\mathcal{I}_{\mathcal{A}}$ be a preferred model of $\Sigma$. We then rewrite the rules in $\Sigma_{S}$ as follows; note that $\alpha_{\left[t_{1} \Rightarrow t_{2}\right]}$ means "in $\alpha$, substitute all occurrences of $t_{1}$ with $t_{2}$ ":

$$
\begin{aligned}
& \Sigma^{\prime} \triangleq\left\{\text { rewrite }\left(\rho_{C}, \operatorname{terms}\left(C, \Sigma_{S}\right)\right) \mid \rho_{C} \in \Sigma_{S}\right\}, \text { with } \\
& \text { rewrite }\left(\rho_{C}, \text { terms }\right) \triangleq C(x) \Rightarrow \\
& \bigwedge_{\alpha \in \tau_{C, \Sigma_{S}}} \alpha \wedge \\
& \bigwedge_{t \in \text { terms }, t \neq o_{C}} \operatorname{hasRoot}(t, x)_{\left[o_{C} \rightarrow x\right]} \wedge \\
& \bigwedge_{t_{1}, t_{2} \in \text { terms }, t_{1} \neq t_{2}} t_{1} \neq t_{2_{\left[o_{C} \rightarrow x\right]}} \wedge \\
& \bigwedge_{t_{1} \in \text { terms }, t_{2} \in\left[t_{1}\right]} t_{1}=t_{2\left[o_{C} \rightarrow x\right]}
\end{aligned}
$$

In addition, we need the following axioms:

$$
\begin{aligned}
& \text { 1. } \Sigma^{\prime} \triangleq \Sigma^{\prime} \cup\left\{C\left(o_{C}\right) \mid C \in \text { concepts }(\Sigma)\right\} \\
& \text { 2. } \Sigma^{\prime} \triangleq \Sigma^{\prime} \cup\left\{o_{C} \neq o_{D} \mid C, D \in \operatorname{concepts}(\Sigma), C \neq D\right\} \\
& \text { 3. } \Sigma^{\prime} \triangleq \Sigma^{\prime} \cup\{\forall x, y, z: \\
& \quad \operatorname{hasRoot}(x, y), \operatorname{hasRoot}(y, z) \Rightarrow \operatorname{hasRoot}(x, z)\} \\
& \text { 4. } \Sigma^{\prime} \triangleq \Sigma^{\prime} \cup\{\forall x, y: \\
& \left.\quad \operatorname{hasRoot}\left(x, o_{C}\right), \operatorname{hasRoot}\left(y, o_{D}\right) \Rightarrow x \neq y\right\}, \\
& \quad \text { for all } C, D \in \operatorname{concepts}(\Sigma), C \neq D .
\end{aligned}
$$

Lemma 2. If $\mathcal{I} \models \Sigma^{\prime}$, then $\mathcal{I}$ is a preferred model for $\Sigma$.

Proof. As $\Sigma^{\prime}$ has been constructed from $\Sigma_{S}$ by adding equality atoms to explicitly represent the co-references with inherited Skolem function successors, which have been identified by the merging rule from a preferred model of $\Sigma$, it is clear that any model of $\Sigma^{\prime}$ will force the same co-references, and hence, satisfy point 3 in Definition 6 . Moreover, point 1 in Definition 10 makes sure that we have non-empty concept models for every concept by requiring an instance, hence satisfying condition 1 in Definition 6. Point 2 in Definition 10 enforces distinctness between those constants, and point 3 declares hasRoot as a transitively closed relation. In combination with the added hasRoot atoms in $\Sigma^{\prime}$, and with the axioms in point 4 of Definition 10, this ensures that condition 2 in Definition 6 is satisfied, requiring that the unique concept models do not overlap (no sharing of participants).

Let us return to our example. For $\Sigma=$ $\{S 1 b, S 23, S 4, S 5\} \quad$ we will get $\Sigma_{S}$ as follows:

$\operatorname{Cell}(x) \Rightarrow$

hasPart $\left(x, f_{1}(x)\right)$, Ribosome $\left(f_{1}(x)\right)$,

hasPart $\left(x, f_{2}(x)\right)$, Chromosome $\left(f_{2}(x)\right)$,

inside $\left(f_{1}(x), f_{0}(x)\right)$, Cytosol $\left(f_{0}(x)\right)$,

pairwise_unequal $\left(\left\{x, f_{0}(x), f_{1}(x), f_{2}(x)\right\}\right)$

EukaryoticCell $(x) \Rightarrow \operatorname{Cell}(x)$

hasPart $\left(x, f_{3}(x)\right)$, Euk.Ribosome $\left(f_{3}(x)\right)$,

hasPart $\left(x, f_{4}(x)\right)$, Euk.Chromosome $\left(f_{4}(x)\right)$,

hasPart $\left(x, f_{5}(x)\right), \operatorname{Nucleus}\left(f_{5}(x)\right)$,

inside $\left(f_{4}(x), f_{5}(x)\right)$,

pairwise_unequal $\left(\left\{x, f_{3}(x), f_{4}(x), f_{5}(x)\right\}\right)$

Cell $\left(o_{\text {Cell }}\right)$, Euk.Cell $\left(o_{\text {Euk.Cell }}\right)$, Ribosome $\left(o_{\text {Ribosome }}\right) \ldots$

If we look at the minimal Herbrand model of $\Sigma_{S}$, we find that the following atoms are satisfied for $o_{E u k . C e l l}$ : hasPart $\left(o_{\text {Euk.Cell }}, f_{1}\left(o_{\text {Euk.Cell }}\right)\right)$,

hasPart $\left(o_{\text {Euk.Cell }}, f_{2}\left(o_{\text {Euk.Cell }}\right)\right)$,

inside $\left(f_{1}\left(o_{\text {Euk.Cell }}\right), f_{0}\left(o_{\text {Euk.Cell }}\right)\right)$,

Ribosome $\left(f_{1}\left(o_{\text {Euk.Cell }}\right)\right)$,

Chromosome $\left(f_{2}\left(o_{\text {Euk.Cell }}\right)\right)$,

$\operatorname{Cytosol}\left(f_{0}\left(o_{\text {Euk.Cell }}\right)\right)$,

hasPart $\left(o_{\text {Euk. Cell }}, f_{3}\left(o_{\text {Euk. Cell }}\right)\right)$,

hasPart $\left(o_{\text {Euk.Cell }}, f_{4}\left(o_{\text {Euk.Cell }}\right)\right)$,

hasPart $\left(o_{\text {Euk. Cell }}, f_{5}\left(o_{\text {Euk. Cell }}\right)\right)$,

inside $\left(f_{4}\left(o_{E u k . C e l l}\right), f_{5}\left(o_{E u k . C e l l}\right)\right)$,

Euk.Ribosome $\left(f_{3}\left(o_{E u k . C e l l}\right)\right)$,

Euk.Chromosome $\left(f_{4}\left(o_{\text {Euk.Cell }}\right)\right)$,

Nucleus $\left(f_{5}\left(o_{\text {Euk.Cell }}\right)\right)$,

Moreover, there are pairwise inequality atoms between $o_{\text {Euk.Cell }}, f_{3}\left(o_{\text {Euk.Cell }}\right), f_{4}\left(o_{\text {Euk.Cell }}\right), f_{5}\left(o_{\text {Euk.Cell }}\right)$ and between $o_{\text {Euk.Cell }}, f_{0}\left(o_{\text {Euk.Cell }}\right), f_{1}\left(o_{\text {Euk.Cell }}\right), f_{2}\left(o_{\text {Euk.Cell }}\right)$.

If we next look at $\mathcal{I}_{\mathcal{A}}$, we will find that $\left[f_{3}\left(o_{\text {Euk. Cell }}\right]\right)=$ $\left[f_{1}\left(o_{\text {Euk.Cell }}\right)\right]=\left\{f_{3}\left(o_{\text {Euk.Cell }}\right), f_{1}\left(o_{\text {Euk.Cell }}\right)\right\}$ holds, and likewise $\left[f_{4}\left(o_{\text {Euk.Cell }}\right]\right)=\left[f_{2}\left(o_{\text {Euk.Cell }}\right)\right]=$ $\left\{f_{2}\left(o_{\text {Euk.Cell }}\right), f_{4}\left(o_{\text {Euk.Cell }}\right)\right\}$. Hence, the desired coreferences have been established, e.g., the from $\mathrm{Cell}$ inherited $\operatorname{Ribosome}\left(f_{1}\left(o_{\text {Euk. Cell }}\right)\right)$ is identified as being co-referential with the "local" Euk.Ribosome $\left(f_{3}\left(o_{E u k . C e l l}\right)\right)$.

The abridged strengthened GSKB $\Sigma^{\prime}$ then looks as follows:

$\operatorname{Cell}(x) \Rightarrow$

hasPart $\left(x, f_{1}(x)\right)$, Ribosome $\left(f_{1}(x)\right)$,

hasPart $\left(x, f_{2}(x)\right)$, Chromosome $\left(f_{2}(x)\right)$,

hasRoot $\left(f_{1}(x), x\right)$, hasRoot $\left(f_{2}(x), x\right)$,

pairwise_unequal $\left(\left\{x, f_{1}(x), f_{2}(x)\right\}\right)$

EukaryoticCell $(x) \Rightarrow \operatorname{Cell}(x)$,

hasPart $\left(x, f_{3}(x)\right)$, Euk.Ribosome $\left(f_{3}(x)\right)$,

hasPart $\left(x, f_{4}(x)\right)$, Euk. Chromosome $\left(f_{4}(x)\right)$,

hasPart $\left(x, f_{5}(x)\right), \operatorname{Nucleus}\left(f_{5}(x)\right)$,

inside $\left(f_{4}(x), f_{5}(x)\right), f_{3}(x)=f_{1}(x), f_{4}(x)=f_{2}(x)$,

hasRoot $\left(f_{3}(x), x\right)$, hasRoot $\left(f_{4}(x), x\right)$,

hasRoot $\left(f_{5}(x), x\right)$,

pairwise_unequal $\left(\left\{x, f_{3}(x), f_{4}(x), f_{5}(x)\right\}\right)$

Ribosome (o Ribosome), Chromosome (o Chromosome)

$\ldots o_{\text {Cell }} \neq o_{\text {Euk. Cell }} \ldots$ (axiom sets 2-4 from Def. 10) 
We claim that we can decide the provenance problem for the strengthened GSKB $\Sigma^{\prime}$ syntactically as follows; also recall that in an admissible $\mathrm{KB}$, the consequents do not contain redundant concept atoms:

Definition 11. Syntactic provenance of atoms in $\Sigma^{\prime}$. In a strengthened $G S K B \Sigma^{\prime}$, for $C \in$ concepts $(\Sigma)$, let $\alpha \in \tau_{C, \Sigma^{\prime}}$ be an atom:

- $\alpha=C(f(x))$ is inherited from $D$ if $D\left(f_{s}(x)\right) \in$ $\tau_{C, \Sigma^{\prime}}$ with $D \in\{C\} \cup$ all_superclasses $\left(C, \Sigma^{\prime}\right)$ and $f^{\prime}\left(f_{s}(x)\right)=f(x) \in \tau_{C, \Sigma^{\prime}}$ with $C\left(f^{\prime}(x)\right) \in$ $\tau_{D, \Sigma^{\prime}}$, and there is no more general class SupD with has_superconcept $(D, S u p D)$ for which this is also the case.

- $\alpha=R\left(f_{1}, f_{2}\right)$ is inherited from $D$ if $D\left(f_{s}(x)\right) \in$ $\tau_{C, \Sigma^{\prime}}$ with $D \in\{C\} \cup$ all_superclasses $\left(C, \Sigma^{\prime}\right)$ and $\left\{f_{1}^{\prime}\left(f_{s}(x)\right)=f_{1}(x), f_{2}^{\prime}\left(f_{s}(x)\right)=f_{2}(x)\right\} \subseteq \tau_{C, \Sigma^{\prime}}$ with $R\left(f_{1}^{\prime}, f_{2}^{\prime}\right) \in \tau_{D, \Sigma^{\prime}}$, and there is no more general class SupD with has_superconcept $(D, S u p D)$ for which this is also the case.

If $\alpha$ is not inherited from some concept, it is called local to $C$.

Looking at the example GSKB $\Sigma^{\prime}$, we see that the atoms hasPart $\left(x, f_{3}(x)\right)$ are inherited from Cell, due to $f_{3}(x)=f_{1}(x)$, and hasPart $\left(x, f_{4}(x)\right)$, due to $f_{4}(x)=f_{2}(x)$. Consequently, hasPart $\left(x, f_{5}(x)\right)$, Nucleus $\left(f_{5}(x)\right)$, inside $\left(f_{4}(x), f_{5}(x)\right)$ are local to EukaryoticCell. Hence, for the original GSKB $\Sigma$, hasPart $\left(x, x_{3}\right)$ and hasPart $\left(x, x_{4}\right)$ were inherited from Cell, and hasPart $\left(x, x_{5}\right), \operatorname{Nucleus}\left(x_{5}\right)$, inside $\left(x_{4}, x_{5}\right)$ are local to EukaryoticCell.

We claim that we can decide the co-reference problem for the strengthened GSKB $\Sigma^{\prime}$ syntactically as follows:

Definition 12. Syntactic co-reference of terms in $\Sigma^{\prime}$. Two terms with $t_{1} \in \operatorname{terms}\left(C, \Sigma^{\prime}\right), t_{2} \in \operatorname{terms}\left(D, \Sigma^{\prime}\right)$ are coreferential, if $t_{1}=t_{2}=x$, or $t_{1}(x)=t_{2}(t) \in \tau_{C, \Sigma^{\prime}}$, or $t_{2}(x)=t_{1}(t) \in \tau_{D, \Sigma^{\prime}}$ (note that $t=x$, or $t=f_{s}(x)$ ).

Looking at the example GSKB $\Sigma^{\prime}$, we see that $f_{3}(x)=f_{1}(x)$ are co-referential and hence the Ribosome in Cell is the same as the Euk.Ribosome in EukaryoticCell, and likewise for the Chromosome due to $f_{4}(x)=f_{2}(x)$.

Lemma 3. Syntactic provenance according to Def. 11 is sound and complete for deciding semantic provenance according to Def. 8. Syntactic co-reference according to Def. 12 is sound and complete for deciding semantic co-reference according to Def. 8.

Proof. Soundness is immediate. Completeness is a straightforward too, as Skolem functions are not shared by different consequents in $\Sigma^{\prime}$, and $\Sigma$ was admissible. Moreover, for two different Skolem functions $f_{i}, f_{j}$, with $i \neq j, f_{i}(t)=f_{j}(t)$ will hold for a certain term $t$ in all models of $\Sigma^{\prime}$ if and only if this was explicitly enforced by an equality atom. Note that this also proves Proposition 3.

We can generalize these results to the original GSKB $\Sigma$ as follows. To check the provenance of $\tau_{C, \Sigma}$ we need to keep track during Skolemization which atom $\alpha^{\prime} \in \tau_{C, \Sigma^{\prime}}$ corresponds to $\alpha$, and check the provenance of $\alpha^{\prime}$ in $\Sigma^{\prime}$. Likewise, to check to co-referentiality of two variables, let $t_{1}$ and $t_{2}$ be its corresponding (Skolem function) terms in the Skolemized versions. Now, $x_{1}$ and $x_{2}$ are co-referential in $\Sigma$ iff $t_{1}$ and $t_{2}$ are co-referential in $\Sigma^{\prime}$. Looking at the example GSKB $\Sigma$, we see that $x_{1}$ from $S 1$ is co-referential with $x_{3}$ in $S 23$ since $f_{3}(x)=f_{1}(x)$ in $\Sigma^{\prime}$, and $x_{2}$ from $S 1$ is co-referential with $x_{4}$ in $S 23$ due to $f_{4}(x)=f_{2}(x)$ in $\Sigma^{\prime}$.

However, given that a GSKB may have more than one strengthened version, "to decide" should be understood in a credulous way here. Only in case a provenance information or co-reference holds in all strengthened GSKBs, this would be a skeptical conclusion; it is clear that all strengthened GSKBs can in principle be constructed, due to finiteness of $\mathcal{I}_{\mathcal{H}}$. We can hence present the main result of this paper as follows:

Corollary 1. Given a strengthened $G S K B \Sigma^{\prime}$, we can decide the provenance and co-reference problem on a syntactic basis. For an admissible (input) GSKB $\Sigma$, we can decide the credulous provenance and credulous co-reference problem $b y$ constructing a strengthened GSKB $\Sigma^{\prime}$ first, and check there. The skeptical provenance and skeptical co-reference problem can be decided by constructing all strengthened GSKBs, and checking if a positive answer holds in all of them.

\section{Implementation \& Evaluation}

For the sake of implementability, the implemented algorithm does not really construct a Skolemized version of $\Sigma$ and neither its Herbrand model. Rather, it works directly on the level of formulas, by considering graph morphisms between concept graphs. The operations performed on these graphs can be understood as operations on the minimal Herbrand model and hence, the given logical framework provides a partial logical reconstruction of what this algorithm is doing.

We have applied the algorithm to the AURA GSKB [Gunning, D. and Chaudhri, V. K. et al., 2010], which currently contains 5662 graph-structured concepts, with a number of 141,909 atoms. The GSKB strengthening algorithm identifies 116,442 of these triples as inherited, which amounts to $\approx 82 \%$. Moreover, 22,667 equality atoms were hypothesized, and 2858 cases of variable specialization in a subconcept were found (e.g., the Chromosome in Cell got specialized to Euk. Chromosome in Eukaryotic Cell). The required runtime is $\approx 15$ hours on a Intel Xeon E5607 $2.2 \mathrm{GHz}$ PC with $8 \mathrm{~GB}$ of RAM running Windows 764 Bit with a 64 Bit Common Lisp implementation. The implementation has $\approx 6100$ lines of code.

The actual deterministic implementation of the nondeterministically specified algorithm requires search in order to maximize the number of inequality assertions. The search space (number of possible individual mergings) is tremendously large, and can only be tackled by employing a large number of search heuristics, including time outs and pruning of the search space.

The achieved progress is good enough from a practical point of view - approx. $82 \%$ of all atoms are identified as being inherited. We have given the strengthened GSKBs to the subject matter experts in the knowledge factory [Chaudhri, V. and Dinesh, N., et al, 2011] for evaluation by spot-checking the provenance of atoms. So far, the results are encouraging, 
and only a small percentage of atoms is falsely given a local provenance, which is caused by the heuristics that prune the search space. The identified co-references were correct to a very large extent. A more precise and complete study should be carried out in the future. Since we do not have reliable ground truths for the provenance and co-reference information (otherwise we wouldn't have to compute it), we cannot automatically check the reconstructed GSKB, i.e., in terms of precision and recall.

\section{Related Work, Conclusions, and Outlook}

The problem of anaphora resolution has been studied in the NLG literature to some extent; for example, [Carpenter, 1994; Cohen, 2007] use default rules to hypothesize equality assertions between variables (NLG referents).

The work of [Chaudhri and Tran, 2012] uses answer set programming (ASP) to add so-called UMap atoms to the GSKB specified as an ASP program. They rely on a complicated axiomatic system encoded as ASP rules to capture the semantics of object-oriented knowledge bases, inheritance etc. The semantics is given by the rules of the ASP program, whereas our approach starts with a notion of desirable models and is hence model-theoretic in nature. Moreover, constants are distinct by definition in ASP programs, so equality needs to be modeled on the meta-level. Scalability of the ASP approach has not been demonstrated yet.

The reasoning system KM [Clark and Porter, 1997] uses a so-called unification mechanism, but the lack of a formal semantics makes it difficult to understand and to debug its inferences. One problem with the approach is that unifications are not represented explicitly as (equality or Umap) atoms in the KM GSKB; instead, unficiations are performed by irreversible GSKB-global substitutions. Retraction and comprehension is very difficult and time consuming, especially in case heuristically generated bad unifications have to be revised. Dealing with the effects of such destructive heuristic unifications was very time consuming in AURA.

It is well-known that the modeling of graph structures is challenging in description logic (DL), as derivations from the tree-model property usually result in decidability problems [Vardi, 1996] which can often only be regained by imposing severe artificial modeling restrictions. Although some progress has been made on modeling graph structures with DLs [Motik et al., 2009], those extensions are still too restricted to be used here. Our experience is that graph structures are central to biology, and approximating them by trees results in coarse models. Our framework allows us to express the graph structures truthfully, but comes with other restrictions, too. To the best of our knowledge, there is no body of work in the DL community that provides answers to the problems addressed in this paper, and we are not aware of any abduction or hypothesization algorithm which has ever successfully been applied to a GSKB of this size.

The AURA system and the actual implementation of the algorithm covers additional expressive means that we have not formally reconstructed yet (transitive, functional, hierarchical relations, number restrictions, and disjointness axioms, cyclical GSKBs, etc.) This is future work.
The strengthened GSKB is also the basis for a couple of AURA knowledge base exports in SILK, ASP, FOPL, and TPTP FOF syntax. We also have an OWL2 translation, in which the graphs are approximated (equality atoms are omitted). These GSKBs can be downloaded from http://www.ai.sri.com/halo/ halobook2010/exported-kb/biokb.html after a license agreement. It is interesting to note that many of our OWL2 exports (we have several variants, in increasing complexity) cannot even be checked for consistency by contemporary DL reasoners within reasonable time bounds. This also rationalizes our work on alternative reasoning methods. Acknowledgements: This work was funded by Vulcan Inc.

\section{References}

[Baader et al., 2003] Franz Baader, Diego Calvanese, Deborah L. McGuinness, Daniele Nardi, and Peter F. PatelSchneider, editors. The Description Logic Handbook: Theory, Implementation, and Applications. Cambridge University Press, 2003.

[Carpenter, 1994] Bob Carpenter. Skeptical and credulous default unification with applications to templates and inheritance. In Inheritance, Defaults and the Lexicon, pages 13-37. Cambridge University Press, 1994.

[Chaudhri and Tran, 2012] Vinay K. Chaudhri and Son Cao Tran. Specifying and reasoning with under-specified knowledge base. In International Conference on Knowledge Representation and Reasoning, 2012.

[Chaudhri, V. and Dinesh, N., et al, 2011] Chaudhri, V. and Dinesh, N., et al. Preliminary steps towards a knowledge factory process. In The Sixth International Conference on Knowledge Capture, 2011.

[Clark and Porter, 1997] Peter Clark and Bruce Porter. Building concept representations from reusable components. In Proceedings of AAAI. AAAI Press, 1997.

[Cohen, 2007] Ariel Cohen. Anaphora resolution as equality by default. In Antnio Horta Branco, editor, Anaphora: Analysis, Algorithms and Applications, 6th Discourse Anaphora and Anaphor Resolution Colloquium, DAARC 2007.

[Gunning, D. and Chaudhri, V. K. et al., 2010] Gunning, D. and Chaudhri, V. K. et al. Project halo update - progress toward digital Aristotle. AI Magazine, Fall 2010.

[Hedman, 2004] Shawn Hedman. A First Course in Logic: An Introduction to Model Theory, Proof Theory, Computability, and Complexity. Oxford Texts in Logic, 2004.

[Motik et al., 2009] Boris Motik, Bernardo Cuenca Grau, Ian Horrocks, and Ulrike Sattler. Representing ontologies using description logics, description graphs, and rules. Artif. Intell., 173(14):1275-1309, September 2009.

[Vardi, 1996] VARDI, M. Y. 1996. Why is modal logic so robustly decidable? In Descriptive Complexity and Finite Models, Proceedings of a DIMACS Workshop, DIMACS Series in Discrete Mathematics and Theoretical Computer Science, vol. 31, 1996. 